\title{
The Features of Polymorphism of Detoxification Phase I and II Genes in Women with Infertility Contaminated with Exogenous Phenol
}

\author{
O.A. Kazakova ${ }^{1, *}$, V.B Alekseev ${ }^{2}$ and O.V. Dolgikh ${ }^{3}$ \\ ${ }^{I}$ Department of immunobiological diagnostic methods Federal Scientific Research Center for Medical and Preventive \\ Health Risk Management Technologies, Perm, Russia \\ ${ }^{2}$ Director of FBSI Federal Scientific Research Center for Medical and Preventive Health Risk Management \\ Technologies, Perm, Russia \\ ${ }^{3}$ Department of immunobiological diagnostic methods Federal Scientific Research Center for Medical and Preventive \\ Health Risk Management Technologies, Perm, Russia \\ *Corresponding author. Email: chakina2011@yandex.ru
}

\begin{abstract}
We conducted a study of fertile women living in Perm, an industrially developed, ecologically unfavorable city located in close proximity to the non-ferrous metallurgy enterprise with population of 40 thousand people. In this area, there is a problem of air pollution by hormone-like xenobiotics (phenols). The issue of the role of candidate genes polymorphism in the development of reproductive disorders is especially for the modern metropolis. The observation group consisted of 37 women with spontaneous abortion, the comparison group was formed by 41 conventionally healthy women. All women live under the aerogenic phenol pollution (more than 1.0 MPC s.w. and m.p). The groups are comparable in terms of age, social status and race. In both groups, the levels of phenol in the blood was detected by the method of capillary gas chromatography on the "Crystal 5000"; polymorphism of genes CYP1A1 rs1048943 Ile462Val, CYP1A1_3 rs4646421 C6310T, GSTA4 rs3756980 T / C, GSTP1 rs1695 Ile105Val, GSTP1 rs1138272 Ala114Val was determined by real-time polymerase chain reaction CFR 96. Statistical data were processed in Statistica 10.0. The groups significantly differed in terms of the level of phenol in the blood, relative to the upper limit of the norm ( $\mathrm{p}<0.05)$. An analysis of the Multiplicative Inheritance Model revealed the A allele of the CYP1A1 rs1048943 Ile462Val gene ( $p<0.05$ ) and the A allele of the GSTP1 rs1695 Ile105Val gene ( $p<0.05)$ as a factor associated with the likelihood of spontaneous abortion under the excessive exogenous estrogenation Phenol.
\end{abstract}

Keywords: genes polymorphism, detoxification, infertility, exogenous phenol

\section{INTRODUCTION}

The state of atmospheric air in large industrial metropolises is one of the main subjects of research. This is not surprising, because over the past ten years, the state of atmospheric air, especially in residential areas, has become more unfavorable for the health. The main sources of pollution are mining enterprises, transport, manufacturing, etc. Emissions contain both widespread and specific substances of the first and second hazard classes.
Perm, located in the east of the East European Plain on both sides of the Kama River banks, has specific meteorological conditions: complicated stagnant air phenomena, frequent inversions, and calm. These phenomena create unfavorable conditions for the concentration of pollutants in the surface air layer.

The problem of miscarriage or loss of pregnancy is of particular relevance in for large cities, where there is an increase in the number of pathologies of the reproductive system among the female population [2-3]. 
The etiology of miscarriage is due to many factors, both internal and external. One of the main predisposing factors is poor quality of the air and drinking water.

Haptenic compounds such as phenols have a negative effect on the pregnancy.

A number of authors [1] studied the effect of phenol on the reproductive health of women and revealed a conjugation of this xenobiotic with the response in the form of spontaneous abortions, threats of termination of pregnancy, as well as complications of pregnancy [1].

In their studies, Gear et al. (2017) revealed estrogenlike properties of phenolic compound, bisphenol A, which manifested its action by participating in the inflammatory pathology of the uterus [12].

The negative effect of phenol on the reproductive system of women can be explained by its hormone-like properties - the ability to replace steroid hormones. An excess of phenol creates a hormonal imbalance that triggers a cascade of reactions involving changes in the hormonal, endocrine and immune systems [7].

For the internal environment, the main predisposing factor is genetic polymorphism, which creates conditions for reproductive disorders.

A large number of studies deal with polymorphism of candidate genes in the development of reproductive disorders leading to miscarriage and loss of pregnancy.

Genes for detoxification, immunoregulation, somatic processes and others are involved in the development of miscarriage.

The main genes for detoxification that can have a negative impact on the development of pregnancy are considered to be the genes of detoxification phases I and II.

Polymorphism of the CYP1A1 gene from the cytochrome CYP450 family, which belongs to the first phase of detoxification of xenobiotics [11, 14], as well as steroid hormones [17], is studied as a predisposing factor in the development of miscarriage [8-10].

The genes of the glutathione s-transferase family belong to the second phase of detoxification [12], and are also considered as candidates for the development of reproductive disorders leading to the loss of pregnancy $[4,5,20]$.

Suryanarayana et al (2004) investigated the relationship between the idiopathic recurrent pregnancy loss (IRPL) and polymorphism of CYP450 and GSTs genes (2004) in Indian women. Using the logistic regression method, they revealed a significant association of IRPB with the allele of the CYP1A1 * 2A gene. For GSTs genes, no significant dependencies were observed [18].
Li J. et al. conducted a meta-analysis (2017) of studies to search for the association of polymorphisms of the CYP450 family with miscarriage and concluded that the rs4646903 polymorphism is associated with the development of this pathology in the Asian population. No obvious relationship was obtained for the rs1048943 polymorphism; the authors refer to the insufficient amount of data and the need for further analysis of this polymorphism [15]

Mortezaee F.T et al. (2014) did not reveal any relationship between polymorphism of the CYP1A1 Ile462Val genes and uterine leiomyoma in Iranian women [16].

A study by Zong C. et al. (2014) did not establish a reliable relationship between the development of recurrent spontaneous abortion in Chinese women and GSTA rs3957357 gene polymorphism [19].

Gordeeva et al. (2016) revealed a relationship between polymorphisms of the GSTs family with abnormalities during pregnancy (miscarriage, premature birth, intrauterine growth retardation, congenital fetal malformations) [6].

Thus, the issue of probable participation of exogenous haptens, including phenol, in the development of reproductive disorders and miscarriage requires further study of the role of candidate gene polymorphism in the additivity of scenarios for the formation of reproductive losses.

The aim of this article is to study features of polymorphism of genes of detoxification phases I and II in women with spontaneous abortion in the first trimester, in conditions of excessive contamination with phenol.

\section{METHODS AND MATERIALS}

The study involved 78 women of reproductive age living in the area polluted with hormone-like xenobiotics of technogenic origin at the level exceeding 1.0 MPC s.c and m.p (phenols).

There were two groups: the "observation" group included 37 women aged $29.65 \pm 1.05$ years with spontaneous abortions in the first trimester. The comparison group included 41 healthy women aged $31.42 \pm 0.91$ years. The groups were comparable in terms of age, ethnicity, material status.

The level of contamination with phenol was assessed by the method of capillary gas chromatography on the Kristall 5000

The frequencies of genotypes of polymorphic genes were assessed: CYP1A1 rs1048943 Ile462Val gene (missense variant), CYP1A1_3 rs4646421 C6310T (intron variant), GSTA4 rs3756980 T / C (intronic variant), GSTP1 rs1695 Ilea10511Val (missense variant), GSTP1 
rs1695 Ile10511Val (missense variant) polymerase chain reaction in real time on the BioRAD CFX96.

The data for were processed in Statistica 10.0 (StatSoft); nominative variables (genotypes) were assesed in SNPstats. The frequency of the genotypes was assessed for compliance with the Hardy-Weindberg Equilibrium Law. The Multiplicative and Additive models of inheritance were used to determine the allele and / or genotype as factors causing the undesirable effects. Differences were considered significant at $p<0.05$.

\section{RESULTS}

The chemical analysis of blood in revealed that the level of phenol contamination in the "observation" group exceeds the upper limit of $0.016 \mathrm{mg} / \mathrm{cm}^{3}$ by 3.6 times, $\mathrm{p}<0.05\left(0.058 \pm 0.009 \mathrm{mg} / \mathrm{cm}^{3}\right)$, and in the "comparison" group, the level of contamination is comparable to the reference $\left(0.020 \pm 0.003 \mathrm{mg} / \mathrm{cm}^{3}\right)$. When comparing the levels of contamination with phenol, a significant difference of 2.3 times was found $(t=3.72 ; \mathrm{p}<0.05$; the Shapiro-Wilk test indicated a normal distribution of the phenol level).

Results of the assessment of the frequencies of polymorphisms of detoxification genes are as follows: I phase CYP1A1 rs1048943 Ile462Val and CYP1A1_3 rs4646421 C631T; Phase II GSTA4 rs3756980 T / C, GSTP1rs 1695 Ile105Val, GSTP1 rs1138272
Ala114Val allowed us to determine their compliance with the Hardy-Weindberg equilibrium law (Table 1), which made it possible to conduct further research using the multiplicative model of inheritance.

The comparative frequency distribution for the CYP1A1 gene rs1048943 Ile462Val was characterized by a reduced frequency of the heterozygous genotype in the observation group, which is 4.6 times less than in the comparison group, while in both groups there was no mutant genotype $(\mathrm{p}<0.05)$ (Table 1$)$.

The frequency distribution for the CYP1A1_3 rs 4646421 C631T gene in the observation group is characterized by a predominance of CC homozygote (1.3 times more than in the comparison group), a lower heterozygote frequency (2.6 times less than in the comparison group) and the presence of a mutant homozygotes (absent in the comparison group) (differences are not significant) (Table 1)

The frequency distribution for the heterozygous GSTP1 rs1695 Ile105Val gene in the observation group was 2.1 times lower and for the mutant genotype 2.6 lower $(\mathrm{p}<0.05)($ Table 1$)$.

For polymorphism GSTA4 rs3756980 $\mathrm{T} / \mathrm{C}$ in the observation group, a decrease in the frequency of heterozygous and mutant homozygous genotypes by 2.0 and 2.6 times was observed (the differences are not significant) (Table 1)

Table 1. The frequency distribution of polymorphisms of the genes and their genotypes

\begin{tabular}{|c|c|c|c|c|c|c|c|}
\hline \multirow[t]{2}{*}{ Gene } & \multicolumn{3}{|c|}{ Number, \% } & \multicolumn{2}{|c|}{ Alleles, \% } & \multicolumn{2}{|l|}{ HWE } \\
\hline & Homo 1 & Hetero & Homo 2 & All1 & All2 & $x 2$ & $p$ \\
\hline \multicolumn{8}{|l|}{ Observation } \\
\hline CYP1A1 rs1048943 lle462Val & 0.92 & 0.08 & 0.00 & 0.96 & 0.04 & 0.07 & 0.7972 \\
\hline CYP1A1_3 rs 4646421 C631T & 0.78 & 0.16 & 0.06 & 0.86 & 0.14 & 3.47 & 0.0625 \\
\hline GSTA4 rs3756980 T/C & 0.78 & 0.19 & 0.03 & 0.88 & 0.12 & 0.49 & 0.4860 \\
\hline GSTP1rs 1695 lle105Val & 0.70 & 0.27 & 0.03 & 0.84 & 0.16 & 0.00 & 0.9739 \\
\hline GSTP1 rs1138272 Ala114Val & 0.78 & 0.22 & 0.00 & 0.89 & 0.11 & 0.54 & 0.4609 \\
\hline \multicolumn{8}{|l|}{ Comparison } \\
\hline CYP1A1 rs1048943 lle462Val & 0.63 & 0.37 & 0.00 & 0.82 & 0.18 & 2.06 & 0.1517 \\
\hline CYP1A1_3 rs 4646421 C631T & 0.58 & 0.42 & 0.00 & 0.79 & 0.21 & 0.83 & 0.3620 \\
\hline GSTA4 rs3756980 T/C & 0.54 & 0.38 & 0.08 & 0.73 & 0.27 & 0.01 & 0.9352 \\
\hline GSTP1 rs 1695 lle105Val & 0.34 & 0.58 & 0.08 & 0.63 & 0.38 & 0.72 & 0.3971 \\
\hline GSTP1 rs1138272 Ala114Val & 0.92 & 0.08 & 0.00 & 0.96 & 0.04 & 0.02 & 0.8853 \\
\hline
\end{tabular}

Note: Homo1 is a normal homozygote, Heteroheterozygote, Homo2 is a mutant homozygote, All1 is a normal allele, All2 is a mutant allele. HWEHardy-Weindberg equilibrium. At p>0.05, the equilibrium is not disturbed.

An assessment of the frequency of genotype GSTP1 rs1138272 Ala114Val gene revealed differences between the groups in the heterozygous genotype, with its prevalence in the observation group by 2.7 times, as well as the absence of the mutant homozygous genotype in the groups (differences between the groups were not significant)
The multiplicative model of inheritance made it possible to identify the A allele of the CYP1A1 gene rs1048943 Ile462Val and the A allele of the GSTP1 gene rs1695 Ile105Val as factors associated with reproductive disorders such as spontaneous abortion (p <0.05) (Table 2).

Table 2. Multiplicative inheritance model for the polymorphisms of detoxification genes

\begin{tabular}{|l|l|l|l|l|l|l|l|l|}
\hline \multirow{2}{*}{ Gene (normal allele / mutant allele) } & \multirow{2}{*}{$\mathrm{X} 2$} & \multirow{2}{*}{$\mathrm{N}$} & \multicolumn{4}{|l|}{ Normal allele } & \multicolumn{3}{l|}{ Mutant allele } \\
\cline { 5 - 9 } & & & OR & $\mathrm{Cl}-95$ & $\mathrm{Cl} 95$ & $\mathrm{OR}$ & $\mathrm{Cl}-95$ & $\mathrm{Cl} 95$ \\
\hline CYP1A1 rs1048943 lle462Val (A/G) & 7.73 & 0.0054 & 5.30 & 1.46 & 18.75 & 0.19 & 0.08 & 0.47 \\
\hline
\end{tabular}




\begin{tabular}{|l|l|l|l|l|l|l|l|l|}
\hline CYP1A1_3 rs 4646421 C631T (C/T) & 0.75 & 0.3868 & 1.68 & 0.51 & 5.47 & 0.59 & 0.19 & 1.90 \\
\hline GSTA4 rs3756980 (T/C) & 3.12 & 0.0774 & 2.66 & 0.88 & 7.98 & 0.38 & 0.13 & 1.08 \\
\hline GSTP1rs 1695 lle105Val (A/G) & 4.88 & 0.0272 & 3.10 & 1.10 & 8.57 & 0.32 & 0.12 & 0.85 \\
\hline GSTP1 rs1138272 Ala114Val (C/T) & 1.14 & 0.2858 & 0.33 & 0.04 & 2.76 & 3.03 & 0.35 & 25.54 \\
\hline
\end{tabular}

For the rest of the polymorphisms, the multiplicative model of inheritance did not reveal any significant dependencies. The additive model of inheritance did not identify the genotypes of polymorphisms associated with the development of spontaneous abortion ( $p$ > $0.05)$.

\section{DISCUSSION}

The studies of the individual genetic characteristics (single nucleotide substitutions) in women are relevant $[4,5,8,10,11,13-16,18,20]$. Of particular interest is an analysis of detoxification genes as candidates for the development of reproductive pathologies in the presence of exogenous simulators of reproductive events $[2,7,13]$.

\section{CONCLUSION}

This study assessed the significance of polymorphism of detoxification genes in women with infertility, excessively contaminated with phenol. It was found that polymorphisms of the CYP1A1 rs1048943 Ile462Val and GSTP1 rs1695 Ile105Val genes act as genetic predictors, and their dominant alleles, burdened by the presence of increased phenol contamination, create the likelihood of infertility. Thus, the gene polymorphisms CYP1A1 rs1048943 Ile462Val and GSTP1 rs1695 Ile105Val can be recommended as indicator genes for early diagnosis of the development of spontaneous abortion under the excessive content of an exogenous simulator of estrogens in the air.

\section{REFERENCES}

[1] S.A. Babanov, I.A. Agarkova, I.S. Lipatov, Yu.V. Tezikov, Occupational damage to reproductive system, Gynecol. 17 (2013) 917-920.

[2] L.A. Balabanova, Environmental and professional factors in the Genesis of reproductive disorders, Pract. Med. 30 (2008) 16.

[3] V.V. Borisov, Causes of disorders and prospects for improving the reproductive health in Russian population, Clin. Lect., Consil. Med. 19(7) 70-75.

[4] O.N. Bespalova, T.E. Ivashchenko, O.A. Tarasenko et al., Association of Glutathione-STransferase Genes Polymorphisms with Placental Insufficiency, J. of obstetr. and wom. Dis. 2 (2006) 25-31.
[5] T.V. Viktorova, G.M. Iskhakova, Association of polymorphic variants of genes of glutathione dependent enzymes with reproductive pathology in women, Probl. of reprod. 4 (2010) 16-19.

[6] L.A. Gordeeva, E.N. Voronina, A.N. Glushkov, Genetic features of xenobiotics metabolism and susceptibility to pathology of pregnancy, Part II, Med. in Kuzbass 15(3) (2016) 3-10.

[7] O.A. Kazakova, O.V. Dolgikh, O.O. Sinitsina, Immune and genetic status of women with reproductive disorders in the conditions of exposed contamination of biological media with phenols, Hyg. and sanit. 99(1) (2020) 90-96.

[8] K.S. Kublinsky, CYP1A1, CYP1A2, SULT1A1 and SULT1E1 allelic polymorphism in case of genital endometriosis, In the world of sci. discov. 1(173) (2016) 36-52.

[9] Endometriosis, In the world of sci. discov. 1(173) (2016) 36-52.

[10] I.N. Noskova, N.V. Artymuk, Ya.L. Masenko, Medical and social aspects of adverse pregnancy outcomes in rural women, Med. in Kuzbass 1 (2008) 65-67.

[11] F.M. Paragulgov, E.A. Sosnova, Genetic polymorphisms of fertility factors and their role in regular pregnancy loss, Wom. health probl. 6(3) (2011) 60-64.

[12] J. Ehlting, B. Hamberger, R. Million-Rousseau, D. Werck, Cytochromes $\mathrm{P} 450$ in phenolic metabolism, Phytochem, Rev., 5(2) (2006) 239-270.

[13] R.B. Gear, S.M. Belcher, Impacts of Bisphenol A and Ethinyl Estradiol on Male and Female CD 1 Mouse, Spleen Sci. Rep. 7 (2017) 856.

[14] R.A. Guneidy, N.A. Meguid, S.S. Abdel Ghany et al., Inter individual variation of normal and Down syndrome glutathione transferase in response to different phenolic compounds, Res. J. of Pharmac., Biol. and Chem. Sci. 8(4) (2017) 184-201.

[15] H.S. Lee, M. Yang, Application of CYP 450 expression for biomonitoring in environmental health, Environ. Health Prev. Med. 13 (2008) 84-93.

[16] J. Li, Y. Chen, S. Mo, D. Nai, Potential Positive Association between Cytochrome P450 1A1 Gene 
Polymorphisms and Recurrent Pregnancy Loss: a Meta Analysism, Ann. Hum. Genet. 81(4) (2017) 161-173.

[17] F.T. Mortezaee, M.H. Chaleshtori, M.A. Tabatabaiefar, Lack of Association Between ESR1 and CYP1A1 Gene Polymorphisms and Susceptibility to Uterine Leiomyoma in Female Patients of Iranian Descent, Cell. J. 16(2) (2014) 225-230.

[18] T. Niwa, N. Murayama, Y. Imagawa, H. Yamazaki, Regioselective hydroxylation of steroid hormones by human cytochromes P450, Drug. Metab. Rev. 47(2) (2015) 89-110.
[19] V. Suryanarayana, M. Deenadayal, L. Singh, Association of CYP1A1 gene polymorphism with recurrent pregnancy loss in the South Indian population, Hum. Reprod. 19(11) (2004) 2648-2652.

[20] C. Zong, Y. Sha, H. Xiang et al., Glutathione S transferase A1 polymorphism and the risk of recurrent spontaneous abortion in Chinese Han population, J. of Assisted Reprod. and Genet. 31 (2014) 379-382.

[21] P.L. Zusterzeel, W.L. Nelen, H.M. Roelofs et al., Polymorphisms in biotransformation enzymes and the risk for recurrent early pregnancy loss, Molec. Hum. Reprod. 6(5) (2000) 474-478. 